\title{
Average Concentration Normalized by Body Mass Index
}

National Cancer Institute

\section{Source}

National Cancer Institute. Average Concentration Normalized by Body Mass Index. NCI

Thesaurus. Code C92367.

AUCT AU divided by TAU and then divided by the body mass index. 